\title{
Sensitivity Analysis of Medium Frequency Transformer Design
}

M. Mogorovic and D. Dujic

This material is posted here with permission of the IEEE. Such permission of the IEEE does not in any way imply IEEE endorsement of any of EPFL's products or services. Internal or personal use of this material is permitted. However, permission to reprint / republish this material for advertising or promotional purposes or for creating new collective works for resale or redistribution must be obtained from the IEEE by writing to pubs-permissions@ieee. org. By choosing to view this document, you agree to all provisions of the copyright laws protecting it. 


\title{
Sensitivity Analysis of Medium Frequency Transformer Design
}

\author{
Marko Mogorovic and Drazen Dujic \\ Power Electronics Laboratory - PEL \\ École Polytechnique Fédérale de Lausanne - EPFL \\ Station 11, CH-1015 Lausanne \\ marko.mogorovic@epfl.ch,drazen.dujic@epfl.ch
}

\begin{abstract}
This paper discusses the technical challenges and trade-offs tied to design of medium frequency transformers (MFTs) for medium-voltage (MV) high-power (HP) power electronic applications, namely emerging solid state transformers (SSTs). A detailed analysis of the factors influencing the MFT operation and limiting the design range is performed. A dedicated MFT design optimization algorithm is used to generate the set of all feasible transformer designs for the given electric requirements, taking into account different MFT geometry ratios, materials and operating frequencies. Design sets are generated for various combinations of design criteria thus exposing the general trends and impact of different design requirements on the feasible design space.
\end{abstract}

\begin{tabular}{lll} 
& \multicolumn{1}{c}{ NomENCLATURE } \\
$A_{p}$ & - & MFT area product \\
$P_{n}$ & - & MFT nominal power \\
$K_{f}$ & - & Excitation waveform coefficient \\
$K_{u}$ & - & Window utilization coefficient \\
$B_{m}$ & - & Peak flux density \\
$J$ & - & Current density \\
$f$ & - & Switching frequency \\
$\Delta$ & - & Penetration ratio
\end{tabular}

\section{INTRODUCTION}

Novel high-power medium-voltage DC-DC converter technologies are needed to support the development of the emerging MVDC grids as well as various traction applications [1]. The most popular solutions recurring in the literature are based on multiple stages of dual active bridge (DAB) or series resonant converter (SRC) topologies, as displayed in Fig. 1, connected in series at the MV side and in parallel at the lowvoltage side. As can be seen in Fig. 1, the central component of any such switched-mode DC-DC power supply topology is the medium frequency transformer (MFT), providing both the galvanic insulation and input-output voltage matching.

The research interest in the area has intensified recently, both in academic and industrial domain, dealing with modeling and optimization [2]-[5] and specific design challenges such as insulation coordination [6] or multi-winding design [7].

In contrast to traditional line frequency transformers (LFTs), normally operating at low grid frequency with sinusoidal voltage and current excitation, MFTs operate on higher

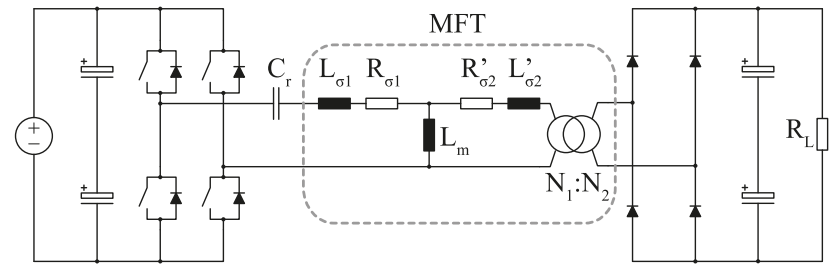

(a)

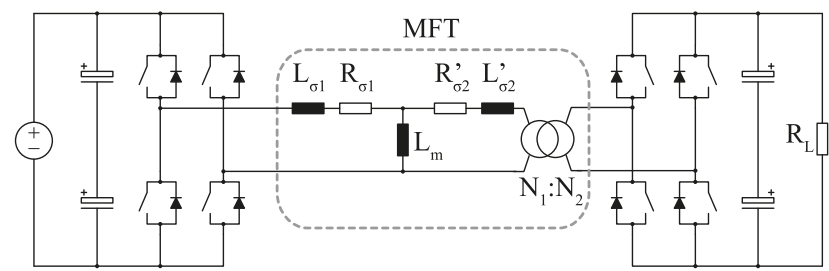

(b)

Fig. 1. DC-DC converter topologies commonly used within SSTs: (a) SRC converter (b) DAB converter

switching frequencies with square voltage and, in general, nonsinusoidal current waveforms characteristic for the given power electronic converter topology. This has implications on MFT losses and dielectric withstand requirements. Moreover, correct design of electric parameters is essential for proper operation of these converters and therefore imposes strict requirements on the accuracy of the corresponding models [8].

The main motivation for operating a transformer at high frequency is the potential for decrease in size, according to approximate relation (1).

$$
A_{p} \approx \frac{P_{n}}{K_{f} K_{u} B_{m} J f}
$$

This has many positive implications: easier integration, less material utilization, lower investment cost and environmental footprint etc. However, size decrease implies decreased cooling surfaces resulting in higher temperature gradients unless additional cooling effort is introduced [9].

These technologies have already been deployed in lowvoltage low-power applications with great success, achieving the expected power densities. However, the increased dielectric withstand and power processing requirements, characteristic for aimed MV applications, affect the maximum achievable power density. Depending on the application requirements, 
TABle I. MFt Scaling Laws For Constant Material Utilisation - Magnetic Flux And CurRent Density

\begin{tabular}{|c|c|c|c|}
\hline Variable & Formula & Proportion & Simplified Generic Shell MFT Structure \\
\hline Cooling Surface & $S_{c}=C_{1} l^{2}$ & $k^{2}$ & \\
\hline Volume and Mass & $M=\gamma V=C_{2} l^{3}$ & $k^{3}$ & \\
\hline Current & $I=J S_{C u}$ & $k^{2}$ & \\
\hline Induced Voltage & $U=C_{3} f B_{m} S_{F e}$ & $f k^{2}$ & \\
\hline Apparent Power & $P=U I$ & $f k^{4}$ & \\
\hline DC Resistance & $R_{D C}=N \rho l / S_{C u}$ & $1 / k$ & \\
\hline Copper Losses & $P_{C u}=F(f) R_{D C} I^{2}$ & $F(f) k^{3}$ & \\
\hline Core Losses & $P_{F e}=K f^{a} B_{m}^{b} V$ & $f^{a} k^{3}$ & \\
\hline Temperature Rise & $\Delta \theta=\left(P_{C u}+P_{F e}\right) /\left(\alpha S_{c}\right)$ & $k\left(F(f)+f^{a}\right)$ & \\
\hline Relative Losses & $P_{r}=\left(P_{C u}+P_{F e}\right) / P$ & $\left(F(f)+f^{a}\right) /(k f)$ & \\
\hline Relative Cost & $\epsilon=M / P$ & $1 /(k f)$ & \\
\hline
\end{tabular}

Where: $l, k$ - spatial dimensions, $C_{i}$ - proportionality constants, $\gamma$ - MFT density, $F(f)$ - skin and proximity effect correction factor

different design choices have direct impact on MFT characteristics.

An MFT is a complex system with coupled multi-physics which makes understanding the effects of certain design changes rather abstract and difficult to grasp. This paper discusses in detail the trends and different design outcomes in both qualitative and quantitative sense. Impact of different parameters is analyzed in a structured and intuitive manner thus exposing different design trade-offs, potential gains and expectation limitations.

\section{Qualitative Analysis of MFT Design TRADE-OFFS}

The approximate relation (1) provides a very simplified estimation of the transformer size, in function of electric requirements and selected design alternatives, suitable for fast design. However, it does not show the effects on the MFT internal characteristics such as temperature gradients and relative cost. A more comprehensive step-by-step qualitative analysis of the scaling laws of each variable characterizing the MFT in mechanical and electric sense, under the assumption of equal material utilization in terms of current and flux densities, is described in Table I.

Starting from basic relations such as calculation of arbitrary MFT surfaces, volume and weight, it is possible to derive the scaling laws for more complex characteristics which are not so intuitively obvious, such as temperature rise, relative losses and relative cost. As can be seen, the relative cost is reverse proportional to both size and frequency. Therefore, from the material quantity and cost point of view, HP MFTs appear more attractive than their smaller counterparts.
However, it can be seen that the temperature rise is proportional to the linear spatial dimension and the sum of the additional winding $(F(f))$ and core $\left(f^{a}\right)$ loss correction factors associated to high frequency effects. Consequently, the temperature gradients increase with the increase of transformer size (processing power), as well as the frequency. Depending on the type of insulation, additional insulation reinforcement may as well substantially increase the thermal resistances towards the ambient (e.g. solid type insulation). Therefore, the frequency, power processing and voltage domain where the described scaling can be preserved without additional cooling effort is limited.

On the other hand, relative losses decrease reversely proportional to size increase, indicating that higher power rated transformers should yield better efficiency. The frequency dependency is a function of winding $(F(f))$ and core $\left(f^{a}\right)$ loss frequency correction factors. The frequency exponent of core materials, $a>1$, and therefore the relative core losses increase with the frequency increase in the amount depending on the material properties. Skin and proximity correction factor $(F(f))$ is negligible for low frequencies $(\Delta<1)$, but increases exponentially at higher frequencies $(\Delta>1)$ [2]. Therefore, relative winding losses have a minimum at some frequency where penetration ratio is around one. Consequently, depending on the core material properties, and loss distribution between the core and the windings, the total relative losses are a convex function of frequency either having a minimum point or an increasing trend in the entire frequency range, depending on the initial gradient.

There are many coupled effects that influence the scaling of the MFT making it a rather difficult task to map the domain of possible designs or fairly assess the design improvement potential of each design choice on its own. 


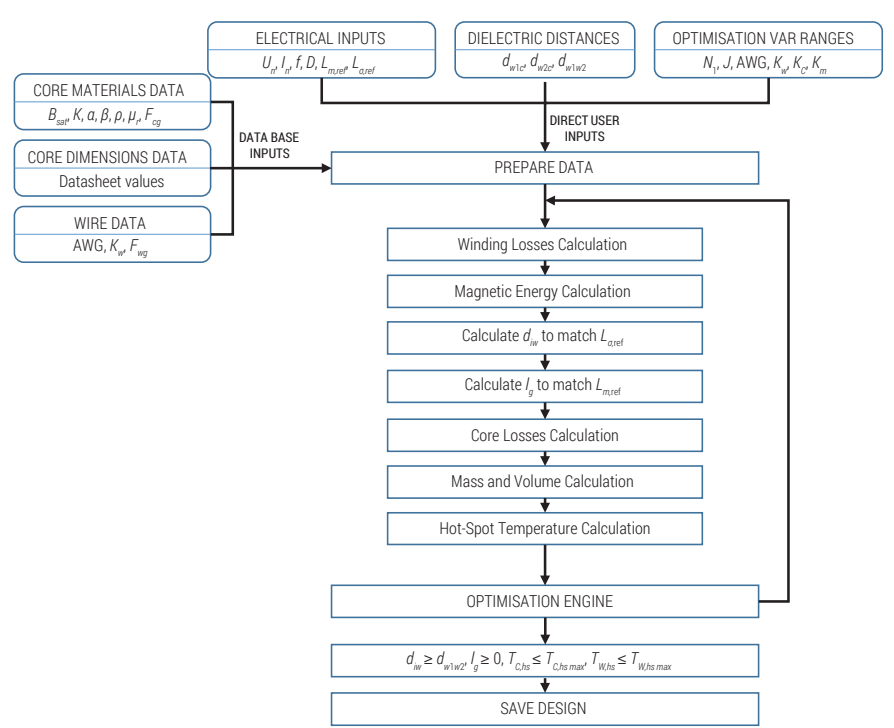

Fig. 2. A brute-force model-based MFT design optimization algorithm [10]

\section{QuAntitative AnAlysis of MFT Design TRADE-OFFS}

This section provides a method of analyzing the MFT design trade-offs in an integral manner using a sophisticated MFT design tool. Without loss of generality, the design is fixed to an air-insulated, air-cooled, N87 Si-ferrite core, AWG 32 litz-wire winding MFT with electrical specifications, as listed in Table II.

TABLE II. MFT PRototype EleCtric SpeCificAtions

\begin{tabular}{|c|c|c|c|c|}
\hline$P_{n}$ & $V_{1}$ & $V_{2}$ & $L_{\sigma 1}, L_{\sigma 2}^{\prime}$ & $L_{m}$ \\
\hline $100 \mathrm{~kW}$ & $750 \mathrm{~V}$ & $750 \mathrm{~V}$ & $4.2 \mu \mathrm{H}$ & $750 \mu \mathrm{H}$ \\
\hline
\end{tabular}

A brute force MFT design optimization algorithm [10], capable of generating sets of all feasible MFT designs for given electric and dielectric specifications, as illustrated in Fig. 2, is used to map various design choice influences and trade-offs. All geometry ratios and relative field densities are considered as optimization variables whose each variation fully defines one MFT design. The maximum allowed hot-spot temperatures of the windings and the core are selected as $150^{\circ} \mathrm{C}$ and $100^{\circ} \mathrm{C}$, respectively, corresponding to the selected material properties.

The feasible MFT design sets are generated for a family of frequencies in range from $1 \mathrm{kHz}$ to $100 \mathrm{kHz}$ in logarithmic scale and displayed as efficiency versus volumetric and weight power density plots, as given in Fig. 3. These plots reveal the trend related to the influence of frequency increase on the disposition of the feasible MFT designs. The upper boundary of the feasibility sets represents the Pareto front corresponding to the trade-off between the efficiency and power density. On the other hand, the lower boundary is set by the thermal limitations, showing how, at the set boundary, a smaller design must have a higher efficiency in order to compensate for the decrease of the cooling surfaces and remain within the defined

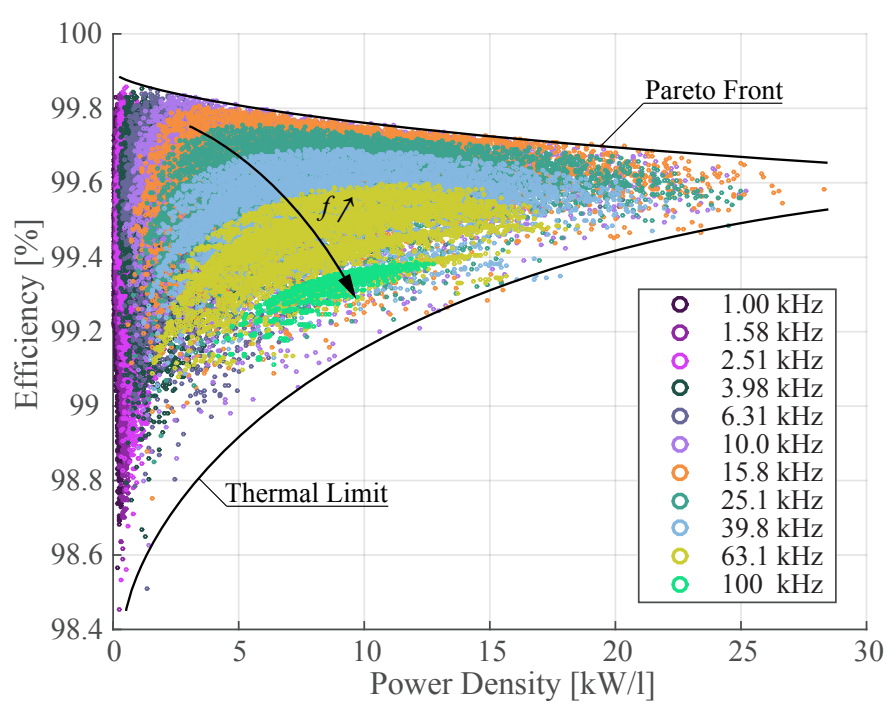

(a)

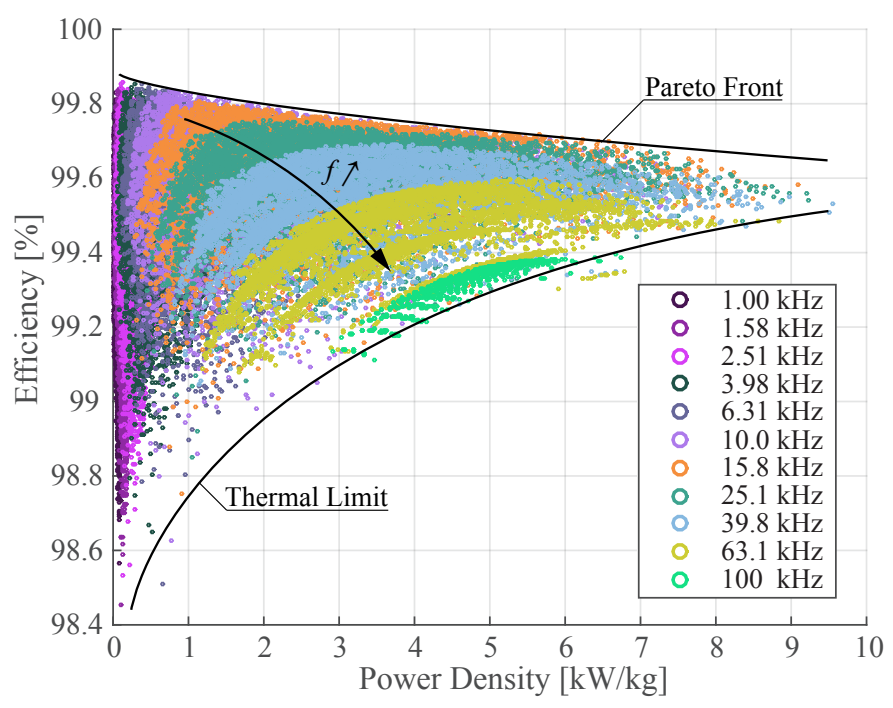

(b)

Fig. 3. Efficiency versus volumetric (a) and weight (b) power density plots of all mathematically feasible MFT designs generated with design optimization algorithm (around 12 million designs, uniformly down-sampled to 66000 for faster rendering). Designs are organized in color groups based on operating frequency

temperature limitations.

It can as well be seen how the feasible design sets at different frequencies reach the maximum achievable efficiency point at different power densities. The loss density within the core and the windings is higher at higher frequencies and therefore the increase of efficiency cannot be achieved by mere increase of cross-sections. Therefore, with the increase of frequency, where additional loss density associated to frequency effects is higher, maximum efficiency points are shifted towards higher power densities. Analyzing only the efficiency versus power density trade-off for any frequency, all of the designs which have lower power density than this critical value are suboptimal as the increase of power density would allow for an increase in efficiency as well. 
Power Density $[\mathrm{kW} / \mathrm{kg}]$
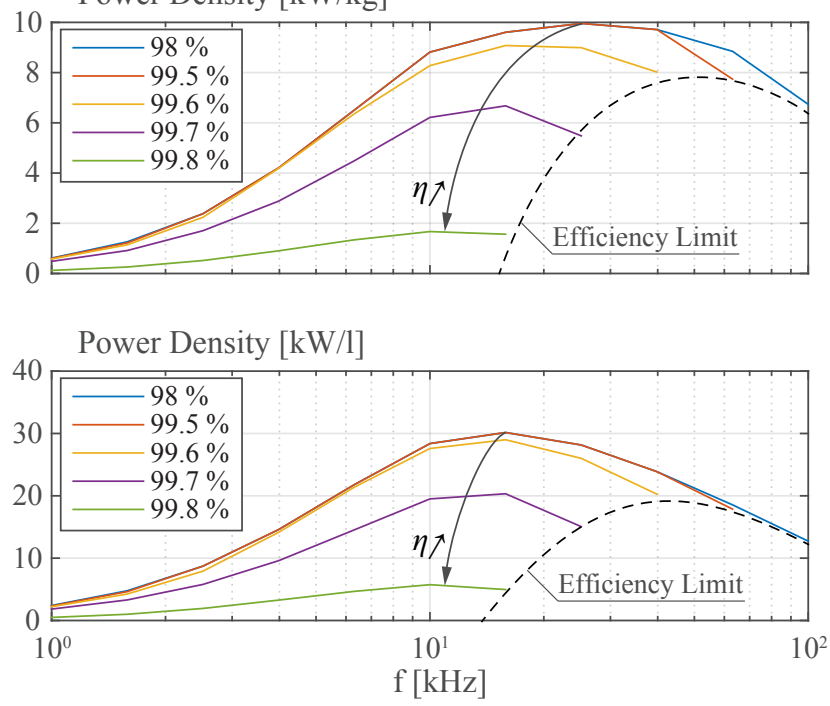

Fig. 4. Maximum achievable weight (top) and volumetric (bottom) power density versus operating frequency plots for different minimum efficiency constraints

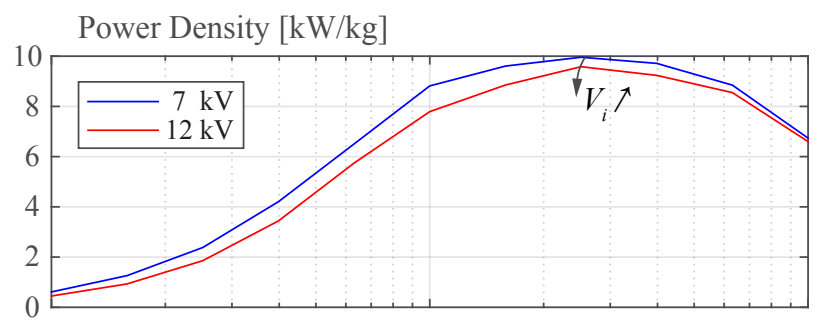

Power Density $[\mathrm{kW} / \mathrm{l}]$

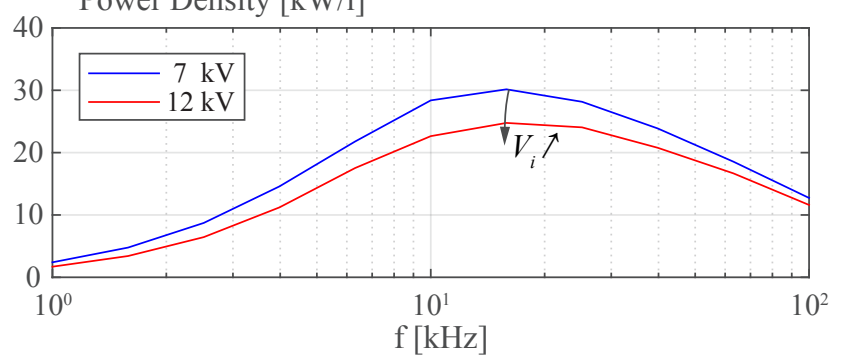

Fig. 5. Maximum achievable weight (top) and volumetric (bottom) power density versus operating frequency plots for different insulation requirements for the secondary winding

Beside these direct conclusions, with some simple postprocessing of these feasible MFT design sets, it is possible to expose all sorts of complex design trends featuring compound constraints.

It is especially interesting to analyze the potential increase in power density that can be achieved with the increase of operating frequency under specified minimum efficiency constraint. This trend can easily be identified by filtering of the maximum MFT design feasibility sets from Fig. 3 by minimum allowed efficiency and selection of designs with maximum power density for each frequency.
The family of curves, representing maximum achievable weight and volumetric power densities versus operating frequency, for different minimum efficiency constraints, are displayed in Fig. 4. It can be seen, that it is not possible to achieve the scaling such as estimated with (1) for higher frequencies. For each minimum efficiency constraint, there exists a Pareto optimal frequency at which the maximum achievable MFT power density is the highest. For higher frequencies, the additional frequency dependent losses start to dominate and it is not possible to maintain the scaling. Furthermore, it can be seen that, as the minimum efficiency constraint is tightened, the maximum achievable power densities decrease, as well as the feasible design set frequency range. It is also interesting to notice that Pareto optimal frequencies are lower for MFT designs with higher efficiency requirement.

On the other hand, MV MFT insulation coordination is not a straight-forward task and it depends on many different details which are not easy to take into account in detail at the optimization stage. However, for design comparison purposes, without the loss of generality, it can be claimed that depending on the chosen insulation material, higher blocking voltage requirements $\left(V_{i}\right)$ will yield larger dielectric distances $\left(d_{i}\right)$, proportional to the corresponding dielectric strength $\left(V_{b}\right)$ according to

$$
d_{i} \approx k_{s} k_{p d} \frac{V_{i}}{V_{b}}
$$

where $k_{p d}$ is a PD test standard voltage front multiplier from IEC 60664-1 international standard and $\left(k_{s}>1\right)$ is a safety margin factor that takes into account the partial discharges and depends on the application and the material.

Therefore, it is possible to perform a simplified analysis of the influence of the required insulation level, by comparing two feasible MFT design sets with two different minimum dielectric distance constraints, corresponding to two different insulation voltage requirements, as displayed in Fig. 5. As can be seen, the maximum achievable power density is lower in case of the MFT design with higher insulation voltage. This is an expected result, as the higher insulation level requires larger dielectric distances and therefore more volume is occupied for this function. Design optimization algorithm optimizes the active part of the transformer, windings and the core, whereas the minimum insulation distances must be respected for a chosen dielectric.

Consequently, dielectric properties of the insulation material determine the sensitivity of the volume and weight of the MFT design to the increase of insulation requirements. Air insulated designs, as featured design choice in this paper, will require large volume to support MV applications, due to relatively poor dielectric properties of air compared to oil or solid, but relatively little added weight, only due to larger mean lengths of the core and the windings, as can be seen in Fig. 5.

Provided that a reliable data base of various insulating materials is available, insulation coordination could as well be integrated into design optimization, thus allowing the selection of the most optimal alternative. 


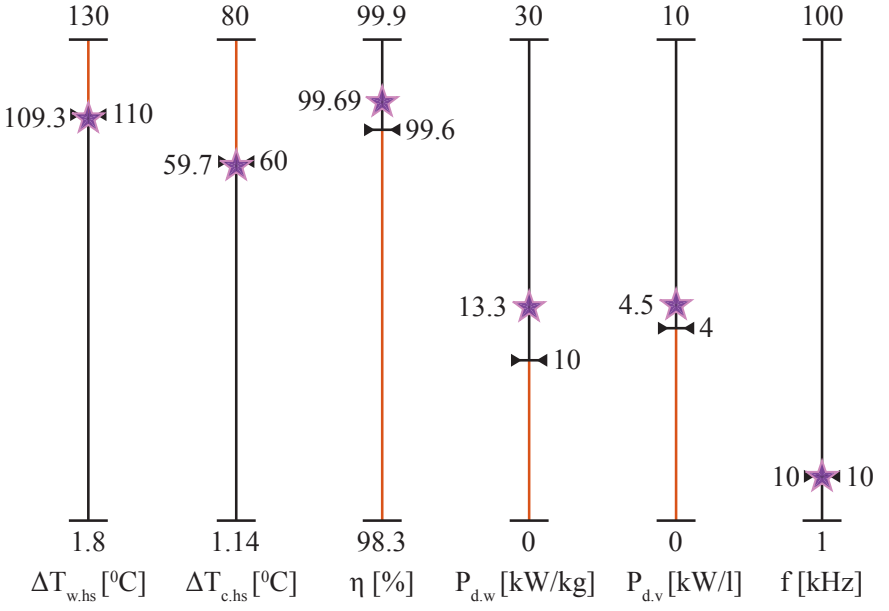

Fig. 6. Position of the optimal $10 \mathrm{kHz}$ MFT design within a multidimensional design space. Scales show the full range of possible MFT characteristics corresponding to the maximum feasibility set at $10 \mathrm{kHz}$ operating frequency. Performance filters, narrowing down the range of each MFT characteristic based on the desired specifications, are specified with black boundary markers and quantified with the value on the right side of each scale. Specifications of the optimal MFT design are depicted with the purple star marker and quantified with the value on the left side of each scale.

\section{MFT PROTOTYPE}

In order to verify the accuracy of the used models and the overall design optimization algorithm, an optimal MFT prototype, for a resonant converter operating at $10 \mathrm{kHz}$, was realized with the aforementioned materials, design choices and electric specifications, as provided in Table II.

The optimal design was selected from the feasible MFT design set for $10 \mathrm{kHz}$ using sophisticated performance filters that allow to arbitrarily narrow down the design ranges based on the desired specifications. The position of the optimal design within a multidimensional design space of interest is displayed in Fig. 6, together with the corresponding filter boundaries. While the discussion about various Pareto optimal fronts is important, as it gives a good insight into the limitations of what is the maximum theoretically achievable performance and provides a good platform for comparison of different combinations of design choices and materials, when it comes to prototyping, it is important to take into account safety margins, especially for phenomena with high stochasticity such as natural convection cooling.

It can be seen that the optimal design is not placed on the boundary of any of the parameter ranges. In order to achieve a thermally robust design, the maximum allowed core and winding hot-spot temperature rise constraint (filter) is tightened by a safety margin of $20^{\circ} \mathrm{C}$, thus generating a new Pareto front between efficiency and power density. The most optimal design is selected on this new Pareto front by adjusting the efficiency and power density filters in a desired manner.

In this way, it is possible to take into account different uncertainties for each MFT characteristic, caused either from limited modeling accuracy or manufacturing and assembly

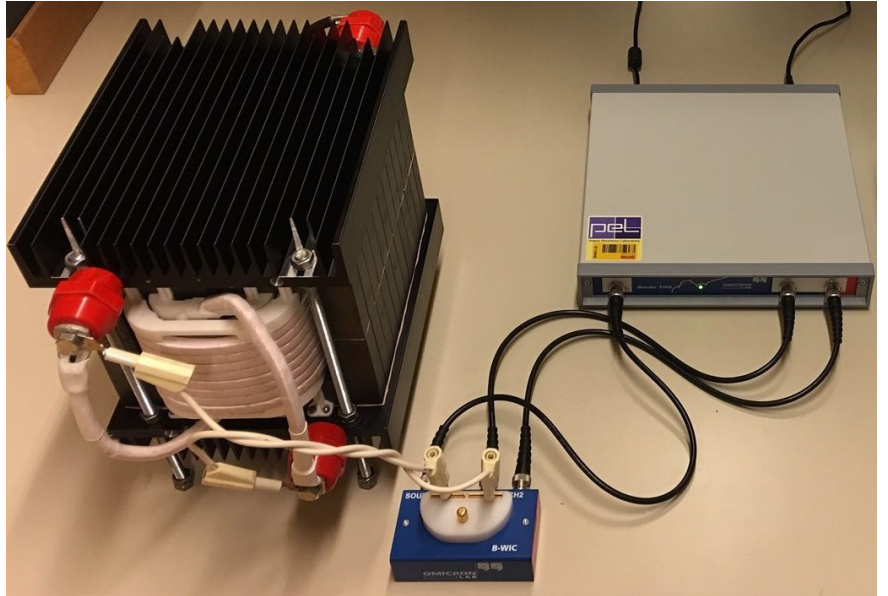

Fig. 7. Measurement of the optimal MFT prototype electric parameters using Bode 100 vector network analyzer

imperfections. The result is a robust Pareto optimal solution that properly takes into account the uncertainty of different models and processes relative to their importance for the given application.

The realized prototype and the electric parameter measurement setup consisting of Bode 100 vector network analyzer are displayed in Fig. 7. The measured electric parameters correlate very well to the reference values, whereas the hot spot temperatures remain within the set safety margins, as described in more detail in [8]-[10].

\section{CONCLUSION}

One of the main drivers for SST operation at medium frequency is the potential to substantially decrease the size of magnetic components. In order to fully benefit from this concept, an MFT design optimization is required to properly take into account all of the effects associated to mediumfrequency high-power operation at MV.

Design optimization of an MFT is a complex task featuring coupled multi-physics and a multitude of different application specific constraints - e.g. electrical parameters, efficiency, temperature rise, weight, volume, height, width etc. A large number of various design choices and materials makes proper classification or comparison of different designs very difficult.

This paper provides a detailed analysis of the key MFT design trends and influences of various factors in a systematic way. A maximum set of feasible MFT designs has been generated for a fixed variation of MFT design choices and a characteristic electrical requirements for resonant converter operation, exposing all the limitations and trade-offs of such design.

By post processing of these maximum feasibility design sets, families of curves, representing various Pareto optimal fronts, are exposed one by one in a systematic manner, providing direct insight into the influence of each optimization variable on the design parameters of interest. This analysis 
provides a great platform for design comparison, exposing the maximum potential gains that can be achieved by manipulation of each optimization variable in relation to others.

However, when it comes to absolute design, modeling and manufacturing uncertainties have to be taken into account properly in relation to the sensitivity of the application to the corresponding MFT parameters. It is shown that, by means of feasible design set filtering, it is possible to set appropriate safety margins for each MFT design property of interest thus offsetting the initial Pareto fronts and generating an optimal robust design.

Finally, even though the analysis has been performed for one fixed combination of design choices and materials, the conclusions can intuitively be extrapolated to other variations in a qualitative sense by appropriately taking into account their relative characteristics.

\section{REFERENCES}

[1] M. Claessens, D. Dujic, F. Canales, J. K. Steinke, P. Stefanutti, and C. Vetterli, "Traction Transformation: A Power-Electronic Traction Transformer (PETT)," ABB Review, No: 1/12, pp. 11-17, 2012.

[2] I. Villar, "Multiphysical Characterization of Medium-Frequency Power Electronic Transformers," PhD thesis, EPFL Lausanne, Switzerland, 2010.

[3] G. Ortiz, "High-Power DC-DC Converter Technologies for Smart Grid and Traction Applications," PhD thesis, ETH Zurich, Switzerland, 2014.
[4] M. Bahmani, "Design and Optimization Considerations of MediumFrequency Power Transformers in High-Power DC-DC Applications," PhD thesis, Chalmers University of Technology Gothenburg, Sweden, 2016.

[5] U. Drofenik, "A 150kW Medium Frequency Transformer Optimized for Maximum Power Density," in 2012 7th International Conference on Integrated Power Electronics Systems (CIPS), Mar. 2012, pp. 1-6.

[6] T. Gradinger, U. Drofenik, and S. Alvarez, "Novel insulation concept for an mv dry-cast medium-frequency transformer," in 19th European Conference on Power Electronics and Applications (EPE'17 ECCE Europe), Warsaw, Poland, 2017., pp. 1-10.

[7] S. Isler, T. Chaudhuri, D. Aguglia, and A. Bonnin, "Development of a $100 \mathrm{~kW}, 12.5 \mathrm{kV}, 22 \mathrm{kHz}$ and $30 \mathrm{kV}$ insulated medium frequency transformer for compact and reliable medium voltage power conversion," in Proceedings of the 19th European Conference on Power Electronics and Applications (EPE 2017 - ECCE Europe), Warsaw, Poland, 2017.

[8] M. Mogorovic and D. Dujic, "Medium frequency transformer leakage inductance modeling and experimental verification," in IEEE Energy Conversion Congress and Exposition (ECCE) 2017, Cincinnatti, OH, USA, 2017., pp. 419-424.

[9] _ - "Thermal modeling and experimental verification of an air cooled medium frequency transformer," in 19th European Conference on Power Electronics and Applications (EPE'17 ECCE Europe), Warsaw, Poland, 2017., pp. 1-10.

[10] _ "Medium frequency transformer design and optimization," in Power Conversion and Intelligent Motion - (PCIM) 2017, Nuremberg, Germany, 2017., pp. 423-430. 